\title{
Lipid Corralling and Poloxamer Squeeze-Out in Membranes
}

\author{
Guohui Wu, ${ }^{1}$ Jaroslaw Majewski, ${ }^{2}$ Canay Ege, ${ }^{1}$ Kristian Kjaer, ${ }^{3}$ Markus Jan Weygand, ${ }^{3}$ and Ka Yee C. Lee ${ }^{1}$ \\ ${ }^{1}$ Department of Chemistry, The Institute for Biophysical Dynamics \& The James Franck Institute, The University of Chicago, \\ Chicago, Illinois 60637, USA \\ ${ }^{2}$ Manuel Lujan, Jr. Neutron Scattering Center, Los Alamos National Laboratory, Los Alamos, New Mexico 87545, USA \\ ${ }^{3}$ Materials Research Department, Risф National Laboratory, DK-4000 Roskilde, Denmark
}

(Received 26 August 2003; published 7 July 2004)

\begin{abstract}
Using $\mathrm{x}$-ray scattering measurements we have quantitatively determined the effect of poloxamer 188 (P188), a polymer known to seal damaged membranes, on the structure of lipid monolayers. P188 selectively inserts into low lipid-density regions of the membrane and "corrals" lipid molecules to pack tightly, leading to unexpected Bragg peaks at low nominal lipid density and inducing lipid/poloxamer phase separation. At tighter lipid packing, the once inserted P188 is squeezed out, allowing the poloxamer to gracefully exit when the membrane integrity is restored.
\end{abstract}

DOI: 10.1103/PhysRevLett.93.028101

Poloxamer is a family of surface-active block copolymers constituted by a hydrophobic poly (propylene oxide) (PPO) moiety capped with hydrophilic poly (ethylene oxide) (PEO) moieties on the two ends, rendering them amphiphilic. Poloxamers are effective in sealing permeabilized cell membranes damaged by disease or trauma, such as electrical shocks and radiation injuries [1-5]; such permeabilization compromises the membrane's barrier function and causes cell death [6,7]. Poloxamer 188 (P188, molecular weight $=8400 \mathrm{~g} / \mathrm{mol}$, with $80 \mathrm{wt} \%$ PEO) was first among its family tested as a membrane sealant due to its wide application in pharmaceuticals with low toxicity [8]. P188 is found to reduce dye leakage from loaded cells after electroporation [1], and can potentially improve cell survival after electroporation used in gene therapy $[9,10]$. Despite poloxamer's importance in restoring membrane integrity, the underlying sealing mechanisms are not well understood. Furthermore, P188 is an efficient gene carrier with gene transfer accomplished via endocytosis [11]. P188 and other poloxamers have also been included in drug delivery systems to sterically stabilize liposomes, prolonging their circulation time [12]. Improvement on poloxamer design for pharmaceutical use clearly relies on a better understanding of poloxamer's interactions with lipid membranes.

To study such interactions, we have used dipalmitoylphosphatidylcholine (DPPC) monolayers to model the outer leaflet of the membrane, and have performed Langmuir isotherm, $\mathrm{x}$-ray reflectivity (XR), and grazing incidence $x$-ray diffraction (GIXD) experiments to address the following: Does P188 seal by selectively inserting into damaged membranes? What is its effect on lipid packing? What is P188's fate when the membrane regains its integrity? The combination of techniques here reveals both macroscopic and molecular details of lipid/poloxamer interactions. In addition, while phase separation between poloxamers and lipids has earlier been postulated [13], our data provide definitive evidence for the
PACS numbers: $87.15 . \mathrm{Kg}, 61.10 . \mathrm{Kw}, 68.03 .-\mathrm{g}, 68.55 . \mathrm{Jk}$

coexistence of poloxamer-rich and poloxamer-poor phases in lipid membranes.

Surface pressure-area $\left(\pi-A_{\mathrm{DPPC}}\right)$ isotherms are collected on a Langmuir trough [14] with a pure water (Millipore, Bedford, MA) subphase at $30^{\circ} \mathrm{C}$. DPPC (Avanti Polar Lipids, Alabaster, AL, USA) monolayers were spread from chloroform solution at a low surface density $(\pi=0 \mathrm{mN} / \mathrm{m}$, area per DPPC molecule, $A_{\text {DPPC }}=107 \AA^{2}$ ). Such a low lipid density mimics a damaged membrane with increased permeability, while an intact membrane is modeled by compressing the film to the bilayer equivalent pressure $(\sim 30-35 \mathrm{mN} / \mathrm{m})[15]$. P188 (BASF, Parisippany, NJ), dissolved in water, is injected into the subphase, giving a concentration of $50 \mu \mathrm{M}$. P188 insertion increases $\pi$ from 0 to $26 \mathrm{mN} / \mathrm{m}$ (Fig. 1) over $40 \mathrm{~min}$, a time chosen to match the helium flushing time needed in $\mathrm{x}$-ray experiments. This increase

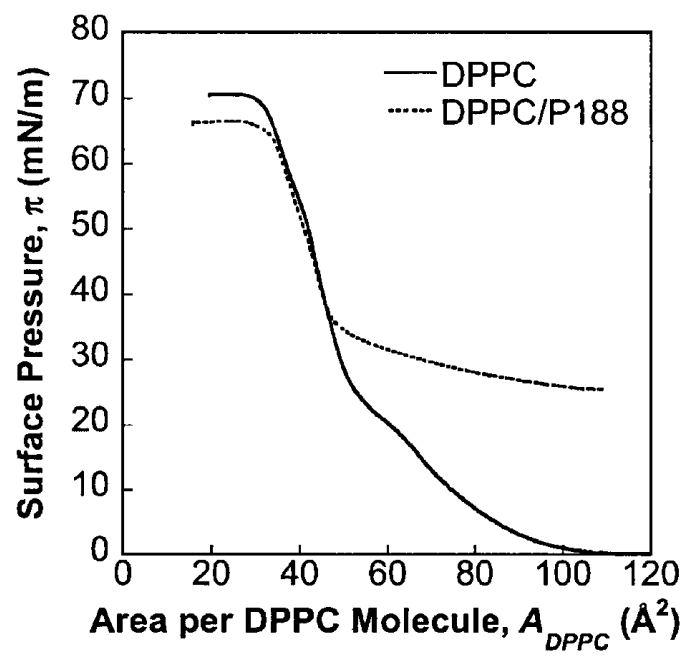

FIG. 1. Isotherms of DPPC (solid) and DPPC/P188 (dashed) on water at $30^{\circ} \mathrm{C}$. At $\pi \geq 36 \mathrm{mN} / \mathrm{m}$, DPPC/P188 and DPPC isotherms overlap, indicating P188 squeeze-out. 
in $\pi$ indicates that P188 migrates readily to the interface at low lipid density. Compression to $\pi>36 \mathrm{mN} / \mathrm{m}$ (dashed line, Fig. 1) reverts the isotherm to that of pure DPPC (solid line, Fig. 1), suggesting that P188 is squeezed out of the lipid film.

To examine P188's effect on lipid packing and to probe its location in the film, GIXD and XR have been performed at the BWl beam line, HASYLAB, Germany, using a surface diffractometer $[16,17]$ with a sealed and temperature-controlled trough under helium.

GIXD is a superior technique for determining in-plane periodic structures [18]. Pure P188 shows no Bragg peak under all conditions (data not shown), indicating that P188 alone is not ordered at the interface. For pure DPPC at $0 \mathrm{mN} / \mathrm{m}\left(A_{\mathrm{DPPC}}=107 \AA^{2}\right)$, again no Bragg peak has been observed. This is not surprising as the film is disordered at this large $A_{\text {DPPC }}$. In fact, pure DPPC only shows Bragg peaks when compressed to a much smaller $A_{\text {DPPC }}$ [Fig. 2(a)]. With P188 present, however, two Bragg peaks are observed at $q_{x y}=1.37$ and $1.46 \AA^{-1}$ even at $A_{\text {DPPC }}=107 \AA^{2}$ [Fig. 2(b)]. The diffraction consists of two low-order reflections, indicating a distortedhexagonal unit cell [19]. The calculated $d$ spacings, 4.59 and $4.30 \AA$, give a unit cell with axes $|a|=|b|=5.09 \AA$, angle $\gamma=116^{\circ}$, and molecular area of $46.7 \AA^{2}$ for the ordered phase. This clearly indicates that P188 induces ordering that would otherwise not exist.

How does P188 enhance surface ordering? Analysis of Bragg peaks at $A_{\mathrm{DPPC}}=107 \AA^{2}$ shows that the unit cell for DPPC/P188 is identical to that of pure DPPC at a much reduced area [21]. The fact that P188 readily migrates to the surface at low lipid density, coupled with this GIXD finding, suggests that P188 occupies regions separate from the lipids, reducing the area and corralling the lipids to pack orderly.

Analysis of Bragg rod profiles corroborates this "corralling" effect. From the full width at half maximum height and positions $\left(q_{z}\right)$ of Bragg rods, the length $\left(L_{c}\right)$ and tilt angle of the coherently scattering alkyl chain can be deduced [22,23]. Both DPPC and P188-treated DPPC films at $\pi \geq 30 \mathrm{mN} / \mathrm{m}$ give the same $L_{c}$ of $19 \pm 1 \AA$, similar tilt angles of $30^{\circ} \pm 1^{\circ}(30 \mathrm{mN} / \mathrm{m})$ and $29^{\circ} \pm 1^{\circ}$ $(40 \mathrm{mN} / \mathrm{m})$ from the surface normal, and azimuthal angles of $\approx 12.2^{\circ}(30 \mathrm{mN} / \mathrm{m})$ and $11.7^{\circ}(40 \mathrm{mN} / \mathrm{m})$ from the nearest neighbor [24]. Such identical parameters show that the peaks in P188-treated films come from ordered DPPC domains.

Should P188 lead to phase separation, one would expect surface inhomogeneity, especially at large P188 area fraction. Indeed, Fig. 3 shows how moving the $x$-ray footprint $\left(2 \times 50 \mathrm{~mm}^{2}\right)$ can result in very different $\mathrm{XR}$ profiles in a DPPC/P188 film at $A_{\mathrm{DPPC}}=107 \AA^{2}$. One location gives a featureless profile [Fig. 3(a)] similar to that of pure P188, and can be fitted with one box [electron density (ED), $\rho=0.36 \pm 0.01 e^{-} / \AA^{3}$ and thickness, $d=$ $29.9 \pm 1.4 \AA$ ]. Another has fringes [Fig. 3(b)] typical of a lipid film and can be fitted with two boxes: (1) DPPC tail/
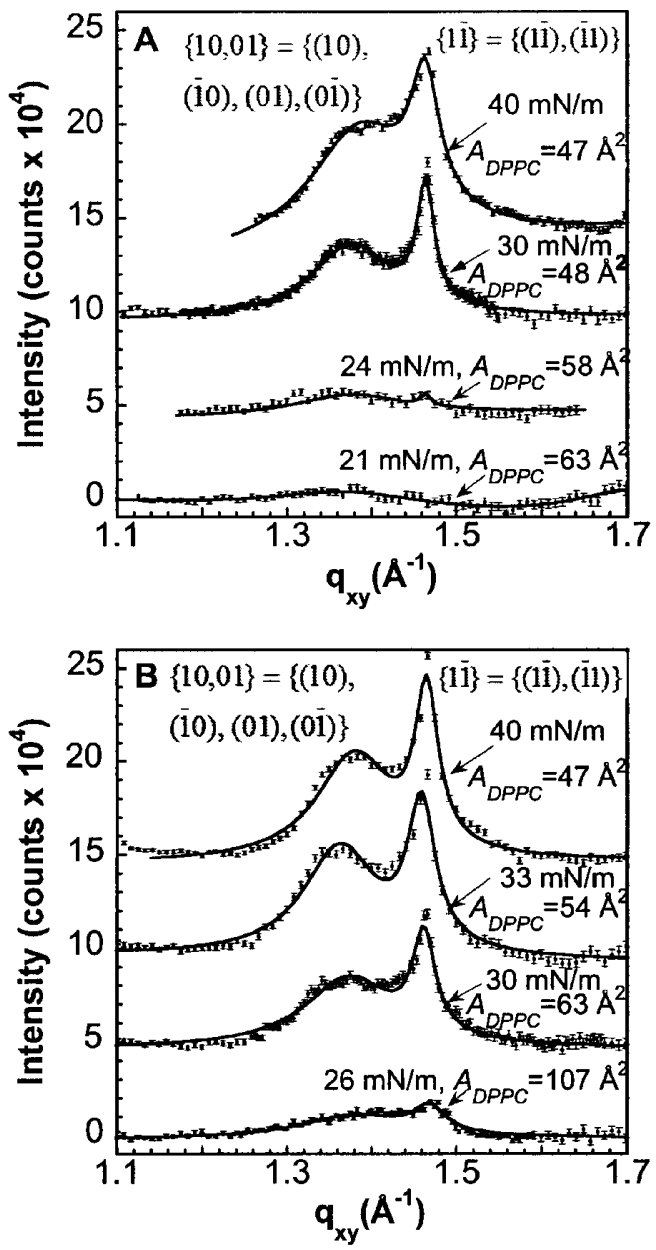

FIG. 2. Bragg peaks from GIXD on water at $30^{\circ} \mathrm{C}$ for (a) DPPC and (b) DPPC/P188. For clarity, the data have been offset vertically. The two Bragg peaks observed for DPPC at $40 \mathrm{mN} / \mathrm{m}$ indicate distorted-hexagonal packing. The Miller indices $\{h, k\}$ are given. In (b), the peak at $A_{\mathrm{DPPC}}=107 \AA^{2}$ corresponds to a highly condensed phase comparable to pure DPPC at $47 \AA^{2} /$ molecule.

P188 $\left(\rho=0.34 \pm 0.01 e^{-} / \AA^{3}, d=14.2 \pm 0.7 \AA\right)$ and (2) DPPC headgroup/P188 $\left(\rho=0.41 \pm 0.01 e^{-} / \AA^{3}, d=\right.$ $9.4 \pm 1.0 \AA ̊)$. Such inhomogeneity persists even when compressed to $A_{\mathrm{DPPC}}=55 \AA^{2}$.

These data, when taken together, show that P188 forces DPPC to form an ordered phase separated from the poloxamer. Phase separation in lipid/poloxamer systems has earlier been inferred [13]. Differential scanning calorimetry has shown that, at high poloxamer concentration, the liposomal bilayer's main phase transition endotherm broadens with a shoulder, indicating two distinct entities present. The presence of mixed micelles suggested by a decrease in mean liposome radius can only indirectly point to separation between poloxamer-rich and poloxamer-poor phases. DPPC ordering by P188 and the presence of surface inhomogeneity observed here unambiguously confirm such phase separation. In the context of membrane sealing, P188 insertion increases the lipid 

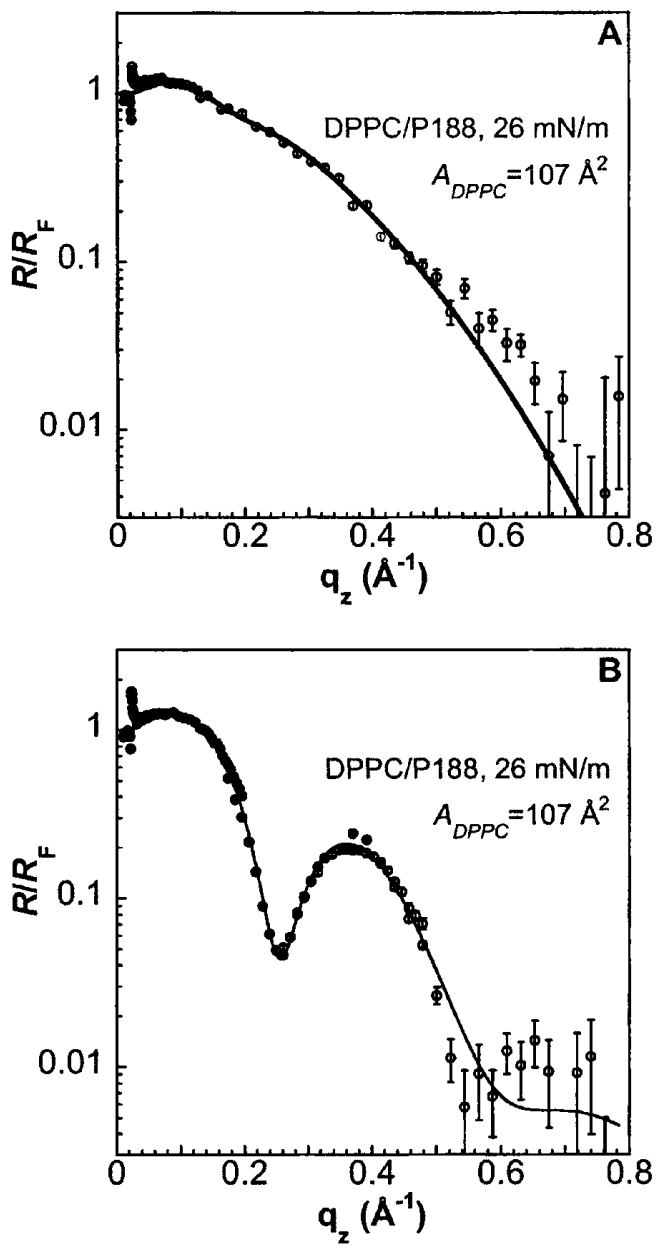

FIG. 3. XR data for DPPC/P188 films on water at $30{ }^{\circ} \mathrm{C}$ for (a) P188-rich and (b) DPPC-rich portions. Solid lines are fit to the data using box models.

packing density, which in turn helps arrest leakage as observed in damaged cells [1]. While the corralling effects of P188 would increase the lipid packing density, we do not expect the lipids to attain the gel state in real membranes due to the large fraction of unsaturated lipids present.

The presence of P188 at the air-water interface at low $\pi$ 's is clearly demonstrated from XR measurements. As the ED contrast between P188 and DPPC headgroup is large (P188's is lower by $\sim 30 \%$ ), $\mathrm{P} 188$ present in the footprint of the beam should reduce the averaged ED of the surface layer in which both the DPPC headgroup and P188 reside. As expected, the best fit gives a significant reduction in the ED of this box $(0.36 \pm 0.01$ and $0.41 \pm 0.01 e^{-} / \AA^{3}$ in Figs. 3(a) and 3(b), respectively, at $26 \mathrm{mN} / \mathrm{m})$ as compared to that for the pure DPPC headgroup $\left(0.44 \pm 0.01 e^{-} / \AA^{3}\right.$ at $24 \mathrm{mN} / \mathrm{m}$ and $0.45 \pm$ $0.01 e^{-} / \AA^{3}$ at $30 \mathrm{mN} / \mathrm{m}$ ), confirming P188's presence in the film. At $26 \mathrm{mN} / \mathrm{m}$ the ED of the DPPC tail region is slightly increased in the presence of P188, signifying the extension of P188 into the layer occupied by the DPPC tail.
What then is the fate of P188 when lipid density is restored? Our x-ray measurements provide quantitative evidence for P188 squeeze-out, which has been qualitatively suggested by isotherm data. XR have been performed for both DPPC (data not shown) and P188treated DPPC films below (Fig. 3) and above (Fig. 4) the "squeeze-out pressure" ( $\left.\pi_{\mathrm{SO}} \approx 36 \mathrm{mN} / \mathrm{m}\right)$, the pressure beyond which both isotherms superimpose. For pure DPPC, compression from 21 to $40 \mathrm{mN} / \mathrm{m}$ results in a thicker hydrocarbon layer $(12.3 \pm 0.3 \rightarrow 16.1 \pm 0.3 \AA$ ), a smaller tilt $\left(49^{\circ} \pm 2^{\circ} \rightarrow 31^{\circ} \pm 2^{\circ}\right)$, and higher ED for the headgroup $\left(0.42 \pm 0.01 \rightarrow 0.46 \pm 0.01 e^{-} / \AA^{3}\right)$ and the tail $\left(0.312 \pm 0.003 \rightarrow 0.322 \pm 0.002 e^{-} / \AA^{3}\right)$.

Upon compression beyond $\pi_{\text {SO }}$ (that is similar to the bilayer equivalent pressure) $\mathrm{XR}$ profiles from pure and P188-treated DPPC film at $40 \mathrm{mN} / \mathrm{m}$ are practically identical [see Fig. 4(a)], confirming the removal of P188 from the film. With the exclusion of P188 above $\pi_{\mathrm{SO}}$, all structural parameters revert to those of pure DPPC and surface inhomogeneities are eliminated.
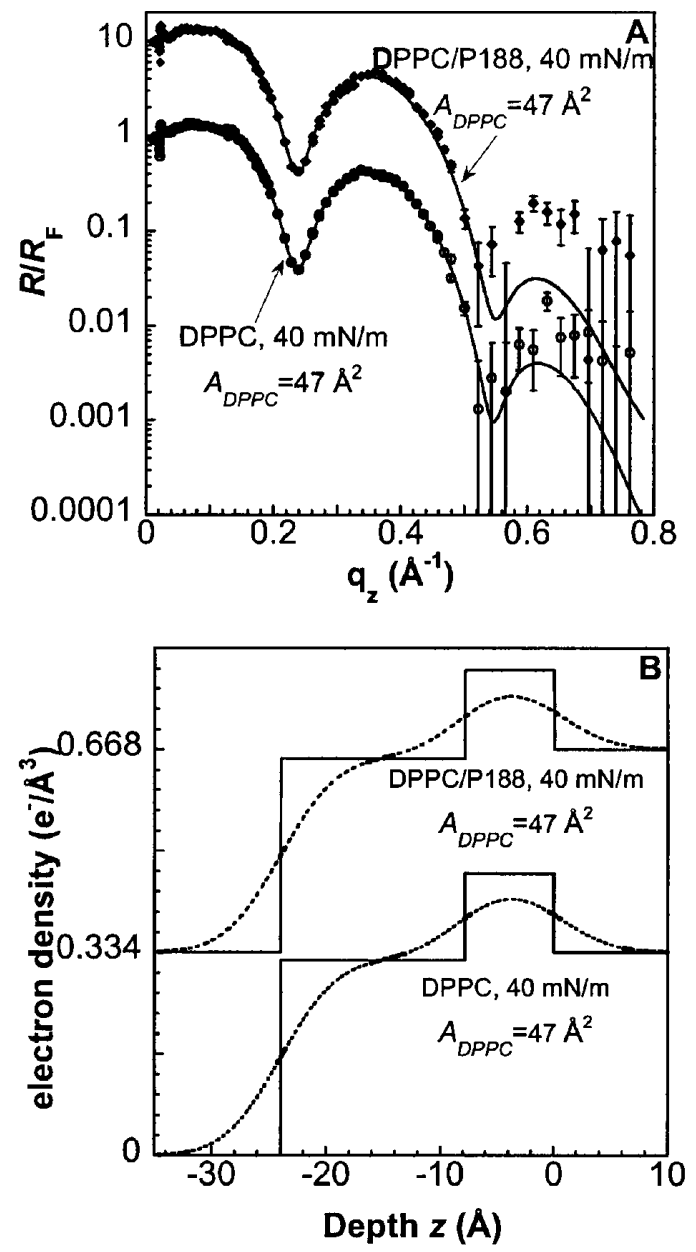

FIG. 4. (a) XR data for DPPC and P188-treated DPPC films on water at $30^{\circ} \mathrm{C}$ for $A_{\mathrm{DPPC}}=47 \AA^{2}$. Solid lines are fits using box models. (b) Corresponding ED profiles. For clarity, the data have been offset vertically 
Further support for squeeze-out comes from GIXD data at $40 \mathrm{mN} / \mathrm{m}$ where the integrated Bragg peak intensity from a P188-treated film is identical to that from pure DPPC. As the integrated intensity is related to the amount of ordering, both films must have a similar fraction of the ordered phase.

P188's selectivity of inserting into low- over highdensity films helps clarify some of the earlier unresolved findings. For instance, it has been reported that the interaction of poloxamers with relatively fluid egg-PC liposomes leads to an increased particle size [28], but such an increase is not seen with gel state liposomes [29]. Castile et al. have found that lipid bilayer pretransition enthalpy is reduced when poloxamer interacts with liquid-crystalline states, but not gel state liposomes [13]. These findings can now be understood in terms of lipid ordering tuning poloxamer insertion, where loose packing in the fluid bilayer promotes insertion while tight packing in the gel state inhibits insertion. Our data further suggest that the poloxamer interacts with the fluid-state membrane by penetrating into, rather than adsorbing onto, bilayer surfaces. Previous work has shown that peptide-lipid interactions investigated utilizing model monolayer and bilayer systems generally yield similar results [30]. While our monolayer work can help shed light on bilayer studies, differences in these two model systems may result in changes in the conformation and orientation of the interacting poloxamer.

P188 inserts when the lipid packing density is low, allowing the damaged membrane to regain barrier control. When the membrane structural integrity is restored, however, P188 is entirely squeezed out from the DPPC film. This remarkable ability of P188 to selectively insert when needed and depart once-damaged membranes after cell healing are major features that promise P188 to be a good therapeutic agent.

We thank R.C. Lee for helpful discussions and J. Kurutz for help with the manuscript. We acknowledge beamtime on BWl at HASYLAB, DESY, Germany, and financial support under the DanSync Program of the Danish Natural Science Research Council. G.W. acknowledges the support of Burroughs Wellcome Fund Interfaces No. 1001774. J. M. was supported by LANL (DOE Contract No. W7405-ENG-36) and the DOE Office of Basic Energy Sciences. C.E was supported by the Alzheimer's Association (IIRG-9901175) and American Health Assistance Foundation (A-1999057). K.Y.C. L. was grateful for support from the Packard (99-1465) and Sloan (BR-4028) Foundations.

[1] R. C. Lee et al., Proc. Natl. Acad. Sci. U.S.A. 89, 4524 (1992).

[2] J. Hannig et al., Radiat. Res. 154, 171 (2000).

[3] D. C. Chang and T. S. Reese, Biophys. J. 58, 1 (1990).
[4] J.T. Padanilam et al., Ann. N.Y. Acad. Sci. 720, 111 (1994).

[5] E. P. Orringer et al., JAMA 286, 2099 (2001).

[6] R. C. Lee, D. Zhang, and J. Hannig, Annu. Rev. Biomed. Eng. 02, 477 (2000).

[7] P. L. McNeil and R. A. Steinhardt, J. Cell Biol. 137, 1 (1997).

[8] I. R. Schmolka, Ann. N.Y. Acad. Sci. 720, 92 (1994).

[9] H. Kusaoke et al., Agric. Biol. Chem. 53, 2441 (1989).

[10] I. T. Agarkova et al., Biologicheskie Membrany 4, 1289 (1987).

[11] J. Liaw, S.-F. Chang, and F.-C. Hsiao, Gene Therapy 8, 999 (2001).

[12] M. C. Woodle, M. S. Newman, and F. J. Martin, Int. J. Pharm. 88, 327 (1992).

[13] J. D. Castile, K. M. G. Taylor, and G. Buckton, Int. J. Pharm. 221, 197 (2001).

[14] A. Gopal and K. Y. C. Lee, J. Phys. Chem. B 105, 10348 (2001).

[15] A. Seelig, Biochim. Biophys. Acta 899, 196 (1987).

[16] J. Majewski et al., Chem. Eur. J. 1, 304 (1995).

[17] I. Weissbuch et al., Adv. Chem. Phys. 102, 39 (1997).

[18] P. Eisenberger and W. C. Marra, Phys. Rev. Lett. 46, 1081 (1981).

[19] The distorted-hexagonal lattice, with $a=b, \gamma \neq 120^{\circ}$, can be thought of as arising through the distortion of a hexagonal lattice in a symmetry direction. It can also be described as a centered rectangular lattice [20]: $a_{r}=$ $2 a \cos \frac{\gamma}{2}, b_{r}=2 a \sin \frac{\gamma}{2}$, and $\gamma_{r}=90^{\circ}$. If a hexagonal lattice is distorted in a nonsymmetry direction, an oblique lattice results.

[20] T. Hahn, International Tables for Crystallography (D. Reidel Publishing Co., Dordrecht, The Netherlands, 1994).

[21] DPPC at $A_{\mathrm{DPPC}}=48 \AA^{2}$ and $30 \mathrm{mN} / \mathrm{m}$ has peaks at $q_{x y}=1.38$ and $1.46 \AA^{-1}$ giving a distorted hexagonal unit cell with $|a|=|b|=5.07 \AA$ and $\gamma=116^{\circ}$.

[22] J. Als-Nielsen and K. Kjaer, in Phase Transitions in Soft Condensed Matter, NATO Advanced Study Institutes, Vol. B211 (Plenum Publishing Corp., New York, 1989), p. 113.

[23] T. R. Jensen and K. Kjaer, in Novel Methods to Study Interfacial Layers, edited by D. Möbius and R. Miller, Studies in Interface Science Vol. 11 (Elsevier, Amsterdam, 2001), p. 205.

[24] Note that the analysis of the Bragg rods (data not shown) indicates that the tilt is in a nonsymmetry direction [25]. Phases with tilt toward nearest neighbor and next nearest neighbor are denoted as $L$ 's in [26] but as phase $I$ and $F$, respectively, in [27].

[25] G. Brezesinski et al., Chem. Phys. Lipids 76, 145 (1995).

[26] I. Kuzmenko, V. M. Kaganer, and L. Leiserowitz, Langmuir 14, 3882 (1998).

[27] D. K. Schwartz, M. L. Schlossman, and P.S. Pershan, J. Chem. Phys. 96, 2356 (1992).

[28] M. Jamshaid et al., Int. J. Pharm. 48, 125 (1988).

[29] S. M. Moghimi et al., Int. J. Pharm. 68, 121 (1991).

[30] L. Silvestro and P. H. Axelsen, Chem. Phys. Lipids 96, 69 (1998). 